\title{
Ein Steuergerechtigkeitskonzept als normativer Maßstab der Untersuchung
}

\subsection{Steuergerechtigkeit durch Gleichbelastung}

Der Begriff "Gerechtigkeit“ ist wie alle Wertbegriffe unbestimmt. Darüber hinaus sind Gerechtigkeitsauffassungen abhängig von Zeit und Raum. ${ }^{48}$

Es ist allgemeine Meinung unter Ethikern, dass der Gleichheitsgrundsatz ein entscheidendes Kennzeichen der Gerechtigkeit ist. ${ }^{49}$ Der englische Philosoph Thomas Hobbes hat die wichtige finanzpsychologische Beobachtung gemacht, dass die Menschen sich weniger durch die Steuerlast als solche, als durch ihre ungleichmäßige Verteilung bedrückt fühlen..$^{50}$ Auch der preußische Staats- und Finanzminister Johannes von Miquel führte in seiner Rede vor dem preußischen Abgeordnetenhaus aus, dass die Ungleichheit der Steuerlasten viel peinlicher sei als die absolute Höhe; ${ }^{51}$ eine Meinung, der sich auch Enno Becker, der Vater der

48 Tipke, Klaus: Steuergerechtigkeit in Theorie und Praxis. Vom politischen Schlagwort zum Rechtsbegriff und zur praktischen Anwendung, Köln 1981, S. 9.

49 Tipke, Klaus: Die Steuerrechtsordnung, S. 284.

50 Hobbes, Thomas: Philosophical Elements of a True Citizen (1658), in: Hobbes Gesamtausgabe, Bd. 2, Aalen 1966, S. $173 \mathrm{f}$.

51 Miquel, Johannes von: Rede zur Rerform der direkten Steuern, Rede vor dem Abgeordnetenhaus am 20.11.1890, S. 305; Pausch, Alfons: Johannes von Miquel. Sein Leben und Werk, Stuttgart 1964, S. 33 . 
Reichsabgabenordnung, anschloss. ${ }^{52}$ In der heutigen Zeit zeigen die Ergebnisse einer empirischen Untersuchung aus dem Jahr 2005, dass die geltenden Steuerprinzipien weitgehend als legitim angesehen werden. Entscheidend für die Bewertung der individuellen Steuerbelastung „scheint zu sein, dass man selbst mit dem eigenen Lebensstandard zufrieden ist und nicht der Eindruck entsteht, andere würden weniger Steuern zahlen als man selbst. " 53

Roman Herzog nannte den Gleichheitssatz „die Magna Charta des Steuerrechts“" ${ }^{\text {"54 }}$ und der deutsche Staatsrechtler und Staatsphilosoph Josef Isensee betont:

Mit der Gleichheit steht und fällt die Steuer. Da sie sich nun einmal nicht aus individuellem Interesse der Betroffenen rechtfertigen läßt, sondern allein aus dem Interesse der Allgemeinheit, akzeptiert sie der Bürger nur, wenn die Last alle nach gleichen Bedingungen trifft. Die allgemeine Lastengleichheit aber wird nur gewährleistet, wenn die jeweilige Steuer nach einsehbaren, konsistenten Prinzipien gesetzlich ausgestaltet ist. $55^{\circ}$

Das Ziel Steuergerechtigkeit ist nur dann zu erreichen, wenn sich der Gesetzgeber in der Ausgestaltung des Steuerrechts Prinzipien und Regeln unterwirft; denn nur dann entsteht ein Steuersystem, ${ }^{56}$ das eine Einheit darstellt und frei von Widersprüchen, Überschneidungen oder Lücken ist. ${ }^{57}$

Eine Analyse dessen, wie sich das Prinzip der Gerechtigkeit im Steuerwesen in den deutschen Staaten seit dem 16. Jahrhundert entwickelt hat, kann nur durchgeführt werden, wenn sie durch eine Wertprämisse geleitet wird. ${ }^{58}$

52 Becker, Enno: Die Reichsabgabenordnung, 7. Aufl., Berlin 1930, S. 162, Anm. 4.

53 Liebig, Stefan/Mau, Steffen: Wann ist ein Steuersystem gerecht? Einstellungen zu allgemeinen Prinzipien der Besteuerung und zur Gerechtigkeit der eigenen Steuerlast, in: Zeitschrift für Soziologie 34 (2005), S. 468-486, S. 468.

54 Herzog, Roman: Steuer- und Finanzpolitik im geeinten Deutschland. Die Sicht des Bundesverfassungsgerichts, in: Bund der Steuerzahler (Hrsg.): Steuer und Finanzpolitik im geeinten Deutschland und Europa, Deutscher Steuerzahler Kongress, Wiesbaden 1991, S. 10-22, S. 11.

55 Isensee, Josef: Vom Beruf unserer Zeit für Steuervereinfachung, in: Steuer und Wirtschaft 71 (1994), S.3-14, S. 3.

56 Hey, Johanna: $\$ 3$ Steuersystem und Steuerverfassungsrecht, in Tipke, Klaus/Lang, Joachim (Hrsg.): Steuerrecht, fortgeführt von Roman Seer u.a., 22. Aufl., Köln 2015, S. 63-137, S. 64.

57 Tipke, Klaus: Steuergerechtigkeit, S. 47.

58 Elschen, Rainer: Steuerliche Gerechtigkeit - Unzulässiger oder unzulänglicher Forschungsgegenstand der Steuerwissenschaften?, in: Steuer und Wirtschaft 65 (1988), S. 1-16, S. 6. 


\subsection{Steuergerechtigkeit durch sachgerechte Prinzipien}

Die Regeln einer Rechtsordnung beruhen nach dem Verständnis der herrschenden Wertungsjurisprudenz auf bestimmten Wertungen, nach denen die Rechtsgemeinschaft ihre Verhältnisse ordnet. ${ }^{59}$ Diese Wertungen bilden das inhaltliche System einer Rechtsordnung, die von Prinzipien als ordnungsstiftenden Grundwerten getragen wird. Diese Rechtsprinzipien sind Ausprägungen einer Leit- oder Rechtsidee ${ }^{60}$ und gebieten als Richtwerte die optimale Verwirklichung des ihnen innewohnenden rechtsethischen Konsenses. ${ }^{61}$ „Fehlt das innere System, die rechtsethische Prinzipienordnung, so ist das Steuerrecht auch keine Gerechtigkeitsordnung. “62

Rechtsprinzipien können in unterschiedlichen Graden erfüllt werden, weil die Ordnung des Rechts pluralistisch zusammengefügt ist. Aus diesem Grunde können die verschiedensten Rechtsprinzipien zusammenwirken, sich ergänzen oder einander

59 „Urteile über Steuergerechtigkeit sind immer Werturteile, die von der subjektiven Wahl des Vergleichsmaßstabs abhängen. "Hundsdoerfer, Jochen: Die einkommensteuerliche Abgrenzung von Einkommenserzielung und Konsum. Eine einzelwirtschaftliche Analyse, Wiesbaden 2002, S. 24. Ohne Besteuerungsgrundsätze, also ohne Werturteile darüber, was steuerliche Gerechtigkeit ist, ist es nicht möglich, Steuerwirkungen zu untersuchen; vgl. Schneider, Dieter: Steuerbilanzen. Rechnungslegung als Messung steuerlicher Leistungsfähigkeit, Wiesbaden 1978, S.21: „Das Steuerrecht ist nicht wirkungs-, sondern wertungsabhängig. "Wagner, Franz W.: Neutralität und GleichmäBigkeit als ökonomische und rechtliche Kriterien steuerlicher Normkritik, in: Steuer und Wirtschaft 69 (1992), S.2-13, S. 7: „Letztlich entspringt jede Begründung für ein Steuersystem einer Wertung. Die der Wertung zugrundeliegenden Axiome sollten erkennbar sein, deren ,Richtigkeit' kann weder bewiesen noch widerlegt werden. "Siegel, Theodor: Konsum- oder einkommensorientierte Besteuerung? Aspekte quantitativer und qualitativer Argumentation, in: Zeitschrift für betriebswirtschaftliche Forschung 52 (2000), S. 724-741, S. 738; vgl. Schmiel, Ute: Werturteilsfreiheit als Postulat für die Betriebswirtschaftliche Steuerlehre?, in: Zeitschrift für betriebswirtschaftliche Forschung 57 (2005), S. 525-545, S. 540; Tipke, Klaus: Die Steuerrechtsordnung, S. 6.

60 Prinzipien ,sind allgemeine Rechtsgrundsätze und Rechtsgedanken, die der Konkretisierung durch Verfassung, Gesetz oder Rechtsprechung bedürfen." Weber-Grellet, Heinrich: Steuern im modernen Verfassungsstaat. Funktionen, Prinzipien und Strukturen des Steuerstaats und des Steuerrechts, Köln 2001, S. 155.

61 Hey, Johanna: $\$ 3$ Steuersystem und Steuerverfassungsrecht, S. 66 f.; Mössner, Jörg Manfred: Prinzipien im Steuerrecht, in: Tipke, Klaus u. a. (Hrsg.): Gestaltung der Steuerrechtsordnung. Festschrift für Joachim Lang zum 70. Geburtstag, Köln 2011, S. 83-99.

62 Lang, Joachim: $\$ 4$ Rechtsstaatliche Ordnung des Steuerrechts, in: Tipke, Klaus/Lang, Joachim (Hrsg.): Steuerrecht, 18. Aufl., Köln 2005, S. 64-130, S.71. Klaus Vogel nennt die Steuerrechtswissenschaft daher auch „Steuergerechtigkeitswissenschaft“, vgl. Vogel, Klaus: Steuerrechtswissenschaft als Steuergerechtigkeitswissenschaft, in: JuristenZeitung 48 (1993), S. 1123. 
widersprechen. Sie haben entweder einen konstruktiven Inhalt (z. B. Konkretisierung des Leistungsfähigkeitsprinzips) oder einen prohibitiven Inhalt (z. B. rechtliche Schranken der Besteuerung) und haben unterschiedlichen Rang und unterschiedliche Wirkkraft. ${ }^{63}$

Ein gerechtes, von der Gesellschaft allgemein anerkanntes Rechtssystem entsteht nur auf der Grundlage von sachgerechten Wertungen. Sachgerechte Wertungen bringen zum einen den rechtsethischen Konsens der Gesellschaft zum Ausdruck, und zum anderen werden sie der Sachlogik des Regelungsgegenstandes gerecht, sie sind daher sachbezogen oder sachangemessen. Bei der Besteuerung ist die ökonomische Rationalität ein bedeutender Faktor der Sachgerechtigkeit; ökonomisch unvernünftige Besteuerungsformen sind meist entsprechend ungerecht. ${ }^{64}$

Unter Ethikern und zeitgenössischen Steuerrechtswissenschaftlern dürfte eine sehr weitgehende Übereinstimmung darüber herrschen, dass von "gerechter“ Besteuerung nur dann die Rede sein kann, wenn den Prinzipien der Allgemeinheit, der Gleichmäßigkeit und der Leistungsfähigkeit Genüge getan ist. Diese drei Prinzipien können jedoch unterschiedlich interpretiert werden. ${ }^{65}$

\subsubsection{Der Grundsatz der Allgemeinheit der Besteuerung}

Der Grundsatz der Allgemeinheit der Besteuerung ${ }^{66}$ ist eine unverzichtbare Grundvoraussetzung einer gerechten Steuerlastverteilung. ${ }^{67}$ Nach diesem Grundsatz sollen alle natürlichen und juristischen Personen,

63 Hey, Johanna: $\$ 3$ Steuersystem und Steuerverfassungsrecht, S. 66 f.

64 Ebd., S.66.

65 Neumark, Fritz: Grundsätze gerechter und ökonomisch rationaler Steuerpolitik, S. 69; Weber-Grellet, Heinrich: Steuern, S. 184; Tipke, Klaus: Die Steuerrechtsordnung, S. 316 ff.

66 Der Grundsatz der Allgemeinheit der Besteuerung wird heute nicht als selbstständiger Grundsatz erfasst, da er Ausfluss des Grundsatzes der Gleichmäßigkeit ist; vgl. z. B. Schneider, Dieter: Steuerbilanzen, S. 16; Hey, Johanna: $\$ 3$ Steuersystem und Steuerverfassungsrecht, S. $66 \mathrm{ff}$. Das Allgemeinheitsprinzip war ein zündendes Losungswort im Kampf gegen den ständischen Staat. Es war vom Zeitalter der Frühen Neuzeit an für das Verständnis der Menschen die wesentliche Voraussetzung einer gerechten Steuerpolitik; denn gegen den Grundsatz, alle Staatsbürger als steuerpflichtig zu betrachten, wurde in der frühen Finanzpolitik massiv verstoßen. Dies betraf insbesondere die Steuerfreiheiten des Klerus und des Adels, Steuerprivilegien, die erst im 19. bzw. im 20. Jahrhundert ihr Ende fanden. Aus der Historie heraus ist daher grundsätzlich eine Trennung notwendig, weshalb die Ausführungen zur Allgemeinheit der Besteuerung am Schluss eines jeden Kapitels gesondert ausgewiesen werden.

67 Meyer, Robert: Die Principien der gerechten Besteuerung in der neueren Finanzwissenschaft, Berlin 1884, S. $301 \mathrm{f}$. 
sofern sie über steuerliche Leistungsfähigkeit verfügen und einer der gesetzlich statuierten Steuerverpflichtungsgründe auf sie zutrifft, ohne Rücksicht auf außerökonomische Kriterien wie (rechtliche) Staatsangehörigkeit, Stand, Klasse, Religion, Rasse usw. zur Steuer herangezogen werden.$^{68}$

Das schließt insbesondere aus, dass z. B. Adel, Klerus, Beamte, Abgeordnete oder andere Staatsträger privilegiert werden; denn die Befreiung Einzelner von steuerlichen Lasten bedeutet in der Regel eine Mehrbelastung anderer. Außerdem führen persönliche und sachliche Steuerbefreiungen regelmäßig dazu, dass die Privilegierten Liquiditätsvorteile erhalten, die oft nachteilige Wettbewerbsverzerrungen zur Folge haben. ${ }^{69}$

Ein weiteres im Grundsatz der Allgemeinheit enthaltenes Postulat fordert, dass Ausnahmen von der subjektiven oder objektiven Steuerpflicht nur insoweit zugelassen werden können, „wie das aus gesamtwirtschafts-, sozial-, kultur-, gesundheitspolitischen oder steuertechnischen Gründen als geboten erscheint. ${ }^{\text {"70 }}$

\subsubsection{Der Grundsatz der Gleichmäßigkeit der Besteuerung}

Der allgemeine Gleichheitssat $\mathrm{z}^{71}$ verlangt, dass die Steuerpflichtigen nicht nur rechtlich, sondern auch tatsächlich gleich belastet werden, d.h., dass die Besteuerungsgleichheit aus zwei Komponenten besteht. ${ }^{72}$

Zum Ersten ist der Gesetzgeber verpflichtet, alles unter einen sachgerechten Besteuerungsmaßstab Fallende als Steuersubjekt zu erfassen und grundsätzlich gleichmäßig zu belasten (Rechtsetzungsgleichheit). Auch wenn ein Gesetz diesem Grundsatz voll entspricht, kann es - gewollte oder ungewollte - Ungleichbehandlungen materiell gleicher Fälle enthalten. Eine in jeder Hinsicht hundertprozentige Gleichmäßigkeit der Steuertechnik ist schlechterdings nicht erreichbar. ${ }^{73}$

68 Neumark, Fritz: Grundsätze gerechter und ökonomisch rationaler Steuerpolitik, S. 75.

69 Davidsohn, Lars: Verfassungsrechtliche Würdigung der sozial- und wirtschaftspolitisch orientierten Besteuerungsideale des 19. und 20. Jahrhunderts, Bielefeld 2007, S. 9.

70 Neumark, Fritz: Grundsätze gerechter und ökonomisch rationaler Steuerpolitik, S. 75.

71 Unter dem Gesichtspunkt des hier vertretenen Gleichmäßigkeitspostulats wird nach Neumark (Neumark, Fritz: Grundsätze gerechter und ökonomisch rationaler Steuerpolitik, S. 92) nur die horizontale Gerechtigkeit verstanden; die vertikale Gerechtigkeit bezieht sich auf Probleme, die in Kapitel 2.3 erörtert werden.

72 Weber-Grellet, Heinrich: Steuern, S. 36; Tipke, Klaus: Die Steuerrechtsordnung, S. 5.

73 Neumark, Fritz: Grundsätze gerechter und ökonomisch rationaler Steuerpolitik, S. 91 und S. 99. 
Zum Zweiten müssen die Finanzbehörden die Steuergesetze gleichmäßig anwenden und durchsetzen (Rechtsanwendungsgleichheit). Die Rechtsanwendung muss der Rechtslage entsprechen, diese verfahrensrechtlich garantieren und die Belastungsgleichheit gewährleisten. Wenn sich die Finanzbehörden uneingeschränkt von Zweckmäßigkeitserwägungen oder politischen Gesichtspunkten leiten lassen, so führt dies auch zu einer Verletzung der Gleichmäßigkeit der Besteuerung.

Abgesehen von gewollten Verstößen gegen den Gleichmäßigkeitsgrundsatz, wie z. B. aufgrund religiöser oder rassenpolitischer Motive im sogenannten Dritten Reich, sind in den meisten Fällen Beweggründe wie Effekte steuerlicher Ungleichbehandlung wirtschafts- bzw. sozialpolitischer Natur, ${ }^{74}$ die nicht nur der Forderung nach Gleichmäßigkeit, sondern auch dem Leistungsfähigkeitsprinzip widersprechen.

Das Gleichmäßigkeitspostulat ist einerseits aus dem Gesichtswinkel von Einzelsteuern zu betrachten. Andererseits vermag nur eine sinnvolle Kombination mehrerer Steuerarten wenigstens tendenziell, dank wechselseitiger Kompensation der Auswirkungen der unterschiedlichen Eigenschaften dieser Steuerarten, der Gesamtheit der Postulate zu entsprechen. ${ }^{75}$

Wenn auch eine in jeder Hinsicht hundertprozentige Gleichmäßigkeit der Besteuerung nicht erreichbar ist, so dürfte grundsätzlich die folgende Definition den verschiedenen Aspekten Rechnung tragen:

Gemäß dem Grundsatz der Gleichmäßigkeit der Besteuerung sind Personen, soweit sie zu irgendeiner Steuer herangezogen werden und sich in gleichen oder gleichartigen steuerlich relevanten Verhältnissen befinden, in bezug auf die betreffende Steuer gleich zu behandeln; als logische Konsequenz daraus ergibt sich das Prinzip der steuerlichen Ungleichbehandlung von Personen in ungleichen Verhältnissen. ${ }^{76}$

\subsubsection{Das Prinzip der Besteuerung nach der Leistungsfähigkeit}

Das Leistungsfähigkeitsprinzip fragt nicht danach, was der Steuerpflichtige vom Staat und von den Gemeinden als öffentliche Leistungen erhält, sondern nur danach, was er aus seinem Einkommen beitragen kann, damit der Staat seine Aufgaben erfüllen kann. ${ }^{77}$ Für Unternehmen gilt das Gleiche - bezogen auf den Gewinn -, denn hinter Unternehmen stehen stets natürliche Personen. Infolgedessen ist die

74 Ebd., S.92.

75 Ebd., S. 4.

76 Ebd., S. 120.

77 Tipke, Klaus: Die Steuerrechtsordnung, S. 479. 
Besteuerung der Unternehmen auf die ursprünglich menschenbezogene Geltung des Leistungsfähigkeitsprinzips zurückzuführen. ${ }^{78}$

Das Problem ist, eine allgemeine Bemessungsgrundlage für die Besteuerung nach der Leistungsfähigkeit zu finden, die festzulegende Eigenschaften zur Gleichbehandlung aller Steuerpflichtigen zu verwirklichen erlaubt. Steuerliche Leistungsfähigkeit umfasst unterschiedliche gesellschaftliche Werturteile über eine distributive Gerechtigkeit. Die verschiedenen Begriffsinhalte von steuerlicher Leistungsfähigkeit sind in den vergangenen Jahrhunderten von zahlreichen Autoren im Einzelnen und unterschiedlich als Rechnungszweck erklärt worden. Sie können systematisch geordnet werden; dabei lassen sich vier alternative Bedeutungsinhalte auseinanderhalten: ${ }^{79}$

Zum einen wird steuerliche Leistungsfähigkeit in der Verwirklichung von Mittelerwerb je Abrechnungsperiode „Jahr“ gesehen (dies führt zur Einkommensteuer), eine andere Möglichkeit besteht in der Verwirklichung von Bedürfnisbefriedigung je Jahr oder bezogen auf den gesamten Lebenszeitraum.

Zum anderen bedeutet steuerliche Leistungsfähigkeit die Möglichkeit der Bedürfnisbefriedigung in jedem Jahr, die durch eine Steuer auf das Gesamtvermögen zu erfassen wäre. Alternativ benennt steuerliche Leistungsfähigkeit die Möglichkeit zum Mittelerwerb je Jahr oder für den gesamten Lebenszeitraum.

Ein Steuersystem, das alternative Fassungen von steuerlicher Leistungsfähigkeit vermischt, kann keine Gleichbehandlung aller Steuerpflichtigen erreichen; denn das Vermengen von Alternativen vernichtet die Beurteilungsbasis für einzelne Argumente. ${ }^{80}$

Eine an der Möglichkeit zum Mittelerwerb oder zur Bedürfnisbefriedigung ausgerichtete Besteuerung scheint gegen den Sinn einer normativen Steuerrechtfertigungs- und Steuerbelastungslehre in einer demokratischen Gesellschaftsordnung zu verstoßen. Demzufolge kann steuerliche Leistungsfähigkeit nur auf die „Verwirklichung“ und nicht auf ein „Potenzial“ bezogen werden. ${ }^{81}$

78 Hey, Johanna: $\$ 3$ Steuersystem und Steuerverfassungsrecht, S.77.

79 Schneider, Dieter: Mängel in der ökonomischen Begründung einer Steuerfreiheit für Kapitaleinkünfte, in: Steuer und Wirtschaft 77 (2000), S.421-424, S. 422 f. sowie Ders.: Bezugsgrößen steuerlicher Leistungsfähigkeit und Vermögensbesteuerung, in: Finanzarchiv, N.F. 37 (1979), S. 26-49.

80 Schneider, Dieter: Bezugsgrößen steuerlicher Leistungsfähigkeit und Vermögensbesteuerung, in: Finanzarchiv, N. F. 37 (1979), S. 26-49, S. 48.

81 Ebd., S. 46. 
Steuerliche Leistungsfähigkeit an der verwirklichten Bedürfnisbefriedigung zu messen, lässt sich zurzeit nicht realisieren; es muss daher auf die zweitbeste Lösung, die Besteuerung des verwirklichten Mittelerwerbs zurückgegriffen werden. ${ }^{82}$

Als Maßgrößen steuerlicher Leistungsfähigkeit kommen insbesondere Einkommen, Konsum und Vermögen des Steuerpflichtigen in Betracht. ${ }^{83}$ Nach der herrschenden Meinung in der Betriebswirtschaftlichen Steuerlehre wird die einkommensteuerliche Leistungsfähigkeit eher im verwirklichten Mittelerwerb, gemessen durch das Markteinkommen, abgebildet. ${ }^{84}$ Dabei wird Einkommen

in der Wirtschaftstheorie verstanden als der Betrag, den eine Person während einer Periode höchstens konsumieren darf, ohne daß sie sich am Ende schlechter steht als am Anfang, d.h. ohne ihr Anfangsvermögen zu mindern. Das Einkommen umfaßt also all das, was die Person durch ihre, Unternehmungstätigkeit ${ }^{`}$ während der Periode zusätzlich erwirtschaftet. ${ }^{85}$

Für diese Untersuchung wird die steuerliche Leistungsfähigkeit nach Neumark wie folgt definiert:

Der Grundsatz der Bemessung und Verteilung der Steuerlasten nach der individuellen Leistungsfähigkeit der Pflichtigen gebietet, die den einzelnen insgesamt zufallenden Steuertraglasten so zu gestalten, daß diese unter Berücksichtigung aller für die Steuerfähigkeitsindikatoren bedeutsamen persönlichen Momente das Verhältnis der individuellen Leistungsfähigkeiten widerspiegeln und demgemäß die durch die Besteuerung bewirkten Einbußen der einzelnen an ökonomisch-finanzieller Dispositionskraft als relativ gleich schwer anzusehen sind. ${ }^{86}$

82 Ebd., S. 48.

83 Hundsdoerfer, Jochen: Die einkommensteuerliche Abgrenzung, S.31. Vgl. Schneider, Dieter: Bezugsgrößen, S. 26-49; Wagner, Franz W.: Neutralität und Gleichmäßigkeit, S. 4 .

84 Hundsdoerfer, Jochen: Die einkommensteuerliche Abgrenzung, S. 31 f.; vgl. Schneider, Dieter: Bezugsgrößen, S.43-46; Elschen, Rainer: Steuerliche Gerechtigkeit, S. $11 \mathrm{f}$.

85 Schneider, Dieter: Steuerbilanzen, S. 36.

86 Neumark, Fritz: Grundsätze gerechter und ökonomisch rationaler Steuerpolitik, S. 135. 


\subsection{Das Redistributionspostulat oder der Grundsatz der steuerlichen Umverteilung von Einkommen und Vermögen}

Das Umverteilungspostulat ${ }^{87}$ ist Ausdruck der sozialen Komponente der Gerechtigkeit, der sozialen Steuergerechtigkeit. ${ }^{88}$ Dabei ist abzuwägen, ob das Gemeinwohlinteresse an der Durchsetzung von Lenkungsnormen den Einbruch in die Besteuerung nach der Leistungsfähigkeit zu rechtfertigen vermag. Entsprechend sind die Wirkungen, die mit der Lenkungsmaßnahme erzielt werden sollen, der Intensität der Verletzung des Leistungsfähigkeitsprinzips abwägend gegenüberzustellen. ${ }^{89}$

Dem Gemeinwohl wird gedient, wenn die Lebensgrundlagen des Gemeinwesens und seiner Mitglieder gefestigt, erhalten oder verbessert werden. Daher ist die Durchsetzung einer Lenkungsnorm nur gerechtfertigt, wenn die Abwägung ergibt, dass diese zu einem größeren gesellschaftlichen Nutzen führt als eine reine Besteuerung nach dem Gleichheitssatz. Es kommt jedoch nicht nur auf den Gesamtnutzen an, die Wirkungen müssen die große Mehrheit der Bevölkerung erfassen, und die große Mehrheit der Nichtbegünstigten muss besser stehen als bei reiner steuerlicher Gleichbelastung. ${ }^{90}$

Für diese Untersuchung wird die steuerliche Umverteilung von Einkommen und Vermögen mit Neumark wie folgt definiert:

87 Unter dem Gesichtspunkt des Redistributionspostulates wird nach Neumark (Neumark, Fritz: Grundsätze gerechter und ökonomisch rationaler Steuerpolitik, S. 92) die vertikale Gerechtigkeit verstanden. Unter Berücksichtigung dieser Differenzierung und der geschichtlichen Entwicklung der sozial- und wirtschaftspolitisch orientierten Besteuerungsideale ab Mitte des 19. Jahrhunderts, die das deutsche Steuerrecht auch heute in erheblichem Maße prägen, wird der Grundsatz der steuerlichen Umverteilung von Einkommen und Vermögen als eine Teilfrage der Besteuerung nach der Leistungsfähigkeit gesondert herausgestellt. Dies erfolgt auch im Hinblick auf ein ideologisch veranlasstes, falsches Verständnis des Gleichheitssatzes und des Wesens der Progression; denn auch die Progression muss sich sachlich legitimieren; vgl. Weber-Grellet, Heinrich: Steuern, S. 172.

88 Tipke, Klaus: Die Steuerrechtsordnung, S. 82.

89 Birk, Dieter: Das Leistungsfähigkeitsprinzip als Maßstab der Steuernormen. Ein Beitrag zu den Grundfragen des Verhältnisses Steuerrecht und Verfassungsrecht (Steuerwissenschaft 13), Köln 1983, S. 232 und S. 244; Tipke, Klaus: Die Steuerrechtsordnung, S. 339.

90 Tipke, Klaus: Die Steuerrechtsordnung, S. 340-347; So sind z. B. wohlstandsmehrende Steuervergünstigungen nach dem Bedürfnisprinzip zu rechtfertigen. Derartige Förderungen durch Steuerentlastungen liegen im Interesse der Allgemeinheit, da die Lebensverhältnisse der Familien materiell gesichert und soziale Gegensätze zwischen Arbeitnehmern und Unternehmern abgebaut werden, vgl. Hey, Johanna: $₫ 3$ Steuersystem und Steuerverfassungsrecht, S. 102. 
Gemäß dem Umverteilungspostulat ist die Gesamtsteuerpolitik so zu gestalten, daß die interindividuelle Verteilung der Steuerlasten über das zur Verwirklichung des Leistungsfähigkeitsgrundsatzes erforderliche Maß hinaus einen progressiven Verlauf nimmt, um auf diese Weise eine gewisse, mit den Grundinstitutionen einer Marktwirtschaftsordnung noch zu vereinbarende und nach vorherrschender Auffassung als gerecht angesehene Redistribution von Einkommen und Vermögen zu erreichen, und zwar im Sinne einer Verringerung der Unterschiede in der durch Marktmechanismus und Marktmacht herbeigeführten primären Verteilung. ${ }^{91}$

Dieses Buch wird unter der Creative Commons Namensnennung 4.0 International Lizenz (http://creativecommons.org/licenses/by/4.0/deed.de) veröffentlicht, welche die Nutzung, Vervielfältigung, Bearbeitung, Verbreitung und Wiedergabe in jeglichem Medium und Format erlaubt, sofern Sie den/die ursprünglichen Autor(en) und die Quelle ordnungsgemäß nennen, einen Link zur Creative Commons Lizenz beifügen und angeben, ob Änderungen vorgenommen wurden.

Die in diesem Buch enthaltenen Bilder und sonstiges Drittmaterial unterliegen ebenfalls der genannten Creative Commons Lizenz, sofern sich aus der Abbildungslegende nichts anderes ergibt. Sofern das betreffende Material nicht unter der genannten Creative Commons Lizenz steht und die betreffende Handlung nicht nach gesetzlichen Vorschriften erlaubt ist, ist für die oben aufgeführten Weiterverwendungen des Materials die Einwilligung des jeweiligen Rechteinhabers einzuholen.

91 Neumark, Fritz: Grundsätze. S. 195. 